\section{Infecciones respiratorias bacteriémicas por Neisseria meningitidis serogrupo W}

Reinaldo Rosas, Sebastián Solar, Luisa Durán, Lorena Porte, Luis Miguel Noriega, Luis Thompson, Alejandra Marcotti, Jorge Pérez y Thomas Weitzel

Respiratory infections and bacteremia caused by Neisseria meningitidis serogroup W

N. meningitidis serogroup $\mathrm{W}$ has recently been introduced into Chile. This serogroup has been associated with hypervirulent strains capable of causing outbreaks. Furthermore, there is data suggesting that the spectrum of clinical manifestations varies among different serogroups. Here we describe three cases of community acquired respiratory infections caused by $N$. meningitidis $\mathrm{W}$, which were diagnosed by blood culture during 2013 in our hospital.

Key words: Meningococcal disease, serogroup W, pneumonia.

Palabras clave: Enfermedad meningocóccica, serogrupo W, neumonía.

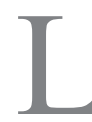
a vigilancia de enfermedad meningocóccica invasora (EMI) en Chile durante el período 2006-2012 ha permitido detectar precozmente la emergencia del serogrupo $\mathrm{W}$ de Neisseria meningitidis por sobre el serogrupo B predominante en los años precedentes ${ }^{1}$. Este fenómeno epidemiológico de introducción de una nueva cepa junto al desplazamiento de la predominante, se pudo observar inicialmente en Sudamérica (Brasil y Argentina $)^{2}$, luego de haberse descrito en Europa, el año 2000, un aumento de los casos de EMI en relación a la peregrinación al "Hajj" en Arabia Saudita $^{3}$. En los meses siguientes a la festividad musulmana ese año, se reportaron más de 400 casos en 16 países, demostrándose con posterioridad la clonalidad genética de una cepa hipervirulenta (ST-11/complejo ET37) ${ }^{4}$. El serogrupo $\mathrm{W}$ se ha asociado en el mundo a prevalencias entre 1 y $8 \%$ del total de casos de EMI, con tasas bajas de portación. No obstante, la emergencia de clones hipervirulentos ha demostrado su capacidad para diseminarse y producir brotes epidémicos secundarios, afectando en Chile con mayores tasas de ataque y mayor letalidad a grupos etarios extremos ${ }^{5}$.

Considerando la emergencia de este nuevo patógeno en Chile, nos parece relevante comunicar la experiencia reciente de tres pacientes adultos hospitalizados durante el 2013 en Clínica Alemana de Santiago. Los pacientes cursaron con meningococcemia por $N$. meningitidis serogrupo W y presentaron una infección respiratoria primaria seguida de rápido deterioro clínico, asociado a una intensa respuesta inflamatoria sistémica. Se trató de una mujer de 57 años con una epiglotitis aguda, un joven de 17 años con una neumonía condensante y un hombre adulto mayor de 91 años con

Departamento de Medicina, Facultad de Medicina, Universidad del Desarrollo (RS, SS, LD, LMN, LT, AM, JP). Laboratorio Clínico, Clínica Alemana de Santiago (LP, TW).

Recibido: 13 de enero de 2015 / Aceptado: 3 de marzo de 2015

Correspondencia a:

Reinaldo Rosas B.

reinaldo.rosas@gmail.com
Tabla 1. Características de pacientes con infecciones respiratorias por N. meningitidis W

\begin{tabular}{|llll|}
\hline & Caso 1 & Caso 2 & Caso 3 \\
Paciente & Mujer, 78 años & Hombre, 98 años & Hombre, 16 años \\
Diagnóstico & Epiglotitis aguda & Neumonía & Neumonía \\
Tiempo de evolución & $48 \mathrm{~h}$ & $24 \mathrm{~h}$ & $72 \mathrm{~h}$ \\
Meningococcemia & Sí & Sí & Sí \\
Meningitis & No & No & No \\
Exantema & No & No & No \\
Radiografía tórax & Atelectasia/ & Consolidación LID & Consolidación LID \\
& derrame & & No \\
Falla renal & Sí & Sí & No \\
Shock & Sí & Sí & No \\
Trombocitopenia & Sí & Sí & $22.600 / \mathrm{mm}^{3}$ \\
\hline Leucocitos ingreso & $13.500 / \mathrm{mm}^{3}$ & $18.600 / \mathrm{mm}^{3}$ & $15 \mathrm{mg} / \mathrm{dL}$ \\
\hline Proteína C-reactiva* & $30 \mathrm{mg} / \mathrm{dL}$ & $29 \mathrm{mg} / \mathrm{dL}$ & Ceftriaxona \\
Tratamiento & Ceftriaxona & Ertapenem, & 10 días \\
Alta & & ceftriaxona & \\
\hline *Valor de referencia: & $0,1-0,5$. & 6 días & \\
\hline
\end{tabular}

una neumonía lobar (Tabla 1). Los tres pacientes tuvieron hemocultivos positivos (BacT/Alert FA, BioMérieux) precozmente en su hospitalización $(15,19$ y $9 \mathrm{~h}$, respectivamente) y ninguno desarrolló compromiso clínico del sistema nervioso central (SNC) ni exantema purpúrico. Las cepas aisladas en cultivo fueron analizadas por espectrometría de masas MALDI-TOF (VITEK MS, BioMérieux) y posteriormente, tipificadas en el Laboratorio de Referencia Nacional (ISP).

Estudios previos han descrito asociaciones de los diferentes serogrupos con presentaciones más septicémicas y con menor compromiso meníngeo $^{6}$. Series clínicas han señalado que las manifestaciones de la EMI por serogrupo $\mathrm{W}$ podrían tener algunas diferencias respecto de las provocadas por los serogrupos $\mathrm{B}$ y C, con menos invasión al SNC y mayor frecuencia de manifestaciones extra-meníngeas asociadas a la meningococcemia ${ }^{7}$. Asimismo, las infecciones respiratorias también han sido reportadas para los serogrupos $\mathrm{W}$ e Y como una presentación clínica de mayor frecuencia a diferencia de otros serogrupos ${ }^{8}$. No obstante, se plantea que el compromiso respiratorio podría verse favorecido por otra infección del tracto respiratorio que provoque las condiciones locales para una invasión de cepas de $N$. meningitidis colonizantes de la vía aérea?

Si este cambio de la epidemiología está afectando actualmente el espectro de manifestaciones clínicas de la EMI en Chile, es desconocido. Sin embargo, se hace necesario dilucidar esta interrogante con la perspectiva de mejorar el diagnóstico precoz en un escenario de incremento de la endemia, secundario a la introducción de un serogrupo emergente. La observación clínica de presentaciones atípicas en Chile donde el predominio del serogrupo $\mathrm{W}$ está impactando con una mayor letalidad a grupos etarios extremos (lactantes menores y adultos mayores), debe ser difundida. En este contexto, parece razonable considerar a la EMI dentro del diagnóstico diferencial de las sepsis severas de foco respiratorio. 


\section{Resumen}

Recientemente se ha descrito la introducción del serogrupo W de Neisseria meningitidis en Chile. La emergencia de este serogrupo se ha asociado con la presencia de cepas hipervirulentas las que tienen la capacidad de diseminarse y producir brotes epidémicos. Además, hay datos que sugieren que existen diferencias en las presentaciones clínicas entre los serogrupos. Presentamos tres pacientes con infecciones respiratorias de la comunidad causadas por $N$. meningitidis $\mathrm{W}$, que fueron diagnosticados por hemocultivos en nuestra clínica durante el año 2013.

\section{Referencias bibliográficas}

1.- Araya P, Díaz J, Seoane M, Fernández J, Terrazas S, Canals A, et al. Vigilancia de laboratorio de enfermedad meningocóccica invasora en Chile, 2006-2012. Rev Chilena Infectol 2014; 31: 377-84.

2.- López E, Debbag R. Enfermedad meningocóccica: siempre presente. Cambios en los serogrupos en el Cono Sur. Rev Chilena Infectol 2012; 29: 587-94.

3.- Mayer L W, Reeves M W, Al-Hamdan N, Sacchi T, Taha M K, Ajello G W, et al. Outbreak of W135 meningococcal diseases in 2000: not emergence of a new W135 strain but clonal expansion within the electophoretic type-37 complex. J Infect Dis 2002; 185: 1596-605.

4.- Popovic T, Sacci C T, Reeves M W, Whitney A M, Mayer L W, Noble C A, et al. Neisseria meningitidis serogroup W135 isolates associated with the ET-37 complex. Emerg Infect Dis 2000; 6: 428-9.

5.- Moreno G, López D, Vergara N, Gallegos D, Advis M, Loayza S. Caracterización clínica de los casos de enfermedad meningocóccica por serogrupo W135 confirmados durante el año 2012 en Chile. Rev Chilena Infectol 2013; 30: 350-60.

6.- Ladhani S N, Beebeejaun K, Lucidarme J, Campbell H, Gray S, Kaczmarski E, et al. Increase in endemic Neisseria meningitidis capsular group W sequence type 11 complex associated with severe invasive disease in England and Wales. Clin Infect Dis 2015; 60: 578-85.

7.- Vienne P, Ducos-Galand M, Guiyoule A, Pires R, Giorgini D, Taha M, et al. The role of particular strains of Neisseria meningitidis in meningococcal arthritis, pericarditis, and pneumonia. Clin Infect Dis 2003; 37: $1639-42$

8.- Cohn A C, MacNeil J R, Harrison L H, Hatcher C, Theodore J, Schmidt M, et al. Changes in Neisseria meningitidis disease epidemiology in the United States, 1998-2007: implications for prevention of meningococcal disease. Clin Infect Dis 2010; 50: 184-91.

9.- Wang J L, Liu D P, Yen J J, Yu C J, Liu H C, Lin C Y, et al. Clinical features and outcome of sporadic serogroup W135 disease Taiwan. BMC Infect Dis 2006; 6: 7 . 